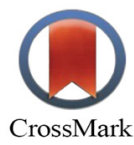

\title{
Pancreatic Tuberculosis in an Immunocompetent Patient: A Case Report and Review of the Literature
}

\author{
Mahsa Abbaszadeh ${ }^{1}$, Jalal Rezai ${ }^{2}$, Mehrdad Hasibi $^{3}$, Mehrdad Larry $^{1, *}$, \\ Mohammad Reza Ostovaneh ${ }^{4}$, Sanam Javidanbardan ${ }^{1}$, Seyed Amir Mirbagheri ${ }^{1}$
}

1. Department of Internal Medicine, Amir Alam Hospital, Tehran University of Medical Sciences, Tehran, Iran

2. Department of Surgery, Amir Alam Hospital, Tehran University of Medical Sciences, Tehran, Iran

3. Department of Infectious Diseases, Amir Alam Hospital, Tehran University of Medical Sciences, Tehran, Iran

4. Division of Gastroenterology, Department of Medicine, Johns Hopkins Medical Institutions, Baltimore, MD, USA

\footnotetext{
* Corresponding Author:

Mehrdad Larry, MD-MPH, Department of Internal medicine, Amir Alam Hospital, Tehran University of Medical Sciences, North Sa'adi Street, 13145-784 Tehran, Iran.

Telefax: + 982166728736

Email: mehrdad.larry@gmail.com
}

Received: 11 May 2017

Accepted: 12 Aug. 2017

\section{ABSTRACT}

Pancreatic tuberculosis is extremely rare and its clinical and radiological findings are similar to those of pancreatic malignancy. The diagnosis of pancreatic tuberculosis is not usually made prior to surgery. Here, we report a case of pancreatic tuberculosis, presented with abdominal pain and weight loss. Abdominal computed tomography (CT) showed a $62 \mathrm{~mm} \times 32 \mathrm{~mm}$ septate solid-cystic mass in the pancreatic head area with extension into the hilum of the liver. There was no evidence of inflammation or pancreatitis. Endoscopic ultrasound-fine needle aspiration (EUSFNA) could not yield the diagnosis. Exploratory laparotomy and further pathological evaluation suggested pancreatic tuberculosis. Response to antituberculosis treatment confirmed the diagnosis. Finally, previous case reports and case studies of pancreatic tuberculosis in the literature are fully investigated.

\section{KEYWORDS:}

Pancreas; Tuberculosis; Biopsy; Fine-Needle; Endosonography

Please cite this paper as:

Abbaszadeh M, Rezai J, Hasibi M, Larry M, Ostovaneh MR, Javidanbardan S, Mirbagheri SA. Pancreatic Tuberculosis in an Immunocompetent Patient: A Case Report and Review of the Literature. Middle East J Dig Dis 2017;9:239-241. doi: 10.15171/mejdd.2017.80.

\section{INTRODUCTION}

Pancreatic tuberculosis is a very rare condition and presents with non-specific symptoms. Radiological imaging shows an incidental abdominal mass. ${ }^{1,2}$ In endemic areas, pancreatic lesions in young individuals and clinical features in favor of tuberculosis (fever and weight loss) should be evaluated for pancreatic tuberculosis. ${ }^{1}$

The diagnosis of pancreatic tuberculosis is often confirmed in exploratory laparotomy; however, in recent years endoscopic ultrasound-fine needle aspiration (EUS-FNA) provides a less invasive diagnostic alternative. Clinical suspicion is important to avoid unnecessary further diagnostic or therapeutic procedures. ${ }^{2}$

\section{CASE REPORT}

A 23-year-old woman presented with epigastric pain and weight loss to Amir Alam hospital, Tehran, Iran. She had lost four kilograms of weight during 2 months but denied any signs of nausea or vomiting and had regular bowel movements. In initial assessment at the emergency room, the patient was afebrile and had stable vital signs. She had marked upper abdominal tenderness but the physical examination was otherwise normal. Her medical or surgical history was not significant. She was not taking any medication and had no drug allergies. 


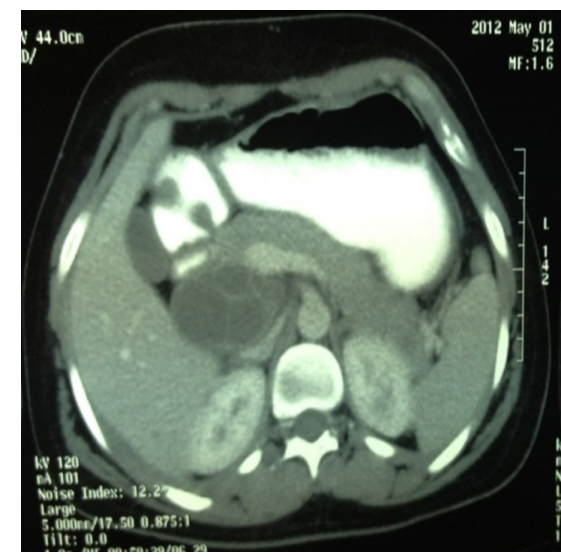

Fig.1: Abdominal tomogram showing a $62 \times 32 \mathrm{~mm}$ septate solid-cystic mass in the pancreatic head area with extension into the hilum of the liver.

Laboratory evaluations revealed mild anemia (hemoglobin level $=11.8 \mathrm{gr} / \mathrm{dL}$ ), increased erythrocyte sedimentation rate $(\mathrm{ESR}=65 \mathrm{~mm} / \mathrm{hr})$ and mild elevation of liver enzymes (aspartate aminotransferase [AST]: $56 \mathrm{IU} / \mathrm{L}$, alanine aminotransferase [ALT]: $67 \mathrm{IU} / \mathrm{L}$, alkaline phosphatase [ALP]: $387 \mathrm{IU} / \mathrm{L}$, and normal amylase: $45 \mathrm{U} / \mathrm{L}$ ). All other tests were normal including fasting blood sugar (FBS): $98 \mathrm{mg} / \mathrm{dL}$, carcinoembryonic antigen (CEA): $1.5 \mathrm{mcg} / \mathrm{L}$, carbohydrate antigen 19 - 9 (CA19 - 9): $12 \mathrm{U} / \mathrm{mL}$, and alpha-fetoprotein (AFP): $3 \mathrm{ng} / \mathrm{mL}$.

Abdominal computed tomography (CT, Bright Speed CT99, GE Medical System) showed a $62 \times 32$ $\mathrm{mm}$ septate solid-cystic mass in the pancreatic head area with extension into the hilum of the liver. There was no evidence of inflammation or pancreatitis (figure 1). EUS (Olympus system) was then performed, which revealed a retroperitoneal mass in favor of retroperitoneal gastrointestinal stromal tumor (GIST). EUS-FNA was performed using $22 \mathrm{G}$ needle and three passes were made. Cytopathological examination ruled out malignancy but was inconclusive. Therefore, the patient underwent exploratory laparotomy. A retroperitoneal cystic lesion was observed in the head of the pancreas and biopsy specimens were taken. The frozen section procedure revealed necrotizing granulomatous infection without malignancy and biopsy specimens were sent for complementary assays. Gram staining, fungal infection staining, and acid-fast bacilli staining were non-contributory but caseating granulomas were observed in pathological examinations. A presumptive diagnosis of pancreatic tuberculosis was made, and isoniazid, rifampin, ethambutol, and pyrazinamide were prescribed for the patient for 6 months. The diagnosis of pancreatic tuberculosis was confirmed in biopsy specimen culture 8 weeks after the surgery.

Six months after starting the treatment, abdominal pain was relieved and the patient started to gain weight. Abdominal CT also revealed complete resolution of the lesion.

\section{DISCUSSION}

Except for a large intrapancreatic inoculum, pancreas tissue resists to mycobacterial invasion. Firstly, due to its unique situation in the retroperitoneum, which is protected from direct environmental exposure. Secondly, this resistance is due to antimycobacterial effects of pancreatic enzymes including lipase and deoxyribonucleases extracts. ${ }^{3}$ Lotfi and colleagues reported only one case in 187 laparotomies in Iran between 1975 and 2004, suggesting no previous case reports of pancreatic tuberculosis from Iran till 2007 to their knowledge ${ }^{1}$

Xia and colleagues described 16 patients from China with pancreatic tuberculosis and peripancreatic lymph nodes. They also reviewed a total of 58 cases of pancreatic tuberculosis, which had been reported in the literature between 1983 and 2003. The predominant symptoms were abdominal pain $(75-100 \%)$, anorexia and weight loss $(69 \%)$, fever and night sweats (50\%), anemia (50\%), and obstructive jaundice (30\%). Laboratory abnormalities including mild anemia, lymphocytopenia, elevated erythrocyte sedimentation rate, and elevated transaminases and alkaline phosphatase have been seen in approximately $50 \%$ of cases. ${ }^{2,4,5}$ Pancreatic mass has been reported as the most common form of pancreatic tuberculosis (more than $50 \%$ of cases) and $70 \%$ of the patients had positive PPD (purified protein derivative) test. ${ }^{5}$ The initial diagnoses included pancreatic tumor, chronic pancreatitis, retroperitoneal tumor, acute pancreatitis, and pancreatic tuberculosis. ${ }^{1}$

Radiographic features in pancreatic tuberculosis are non-specific. Its findings resemble those of pancreatic malignancy. Sonographic imaging shows hypoecho or 
heterogenic masses, and hypodense image on CT. There may be multicystic lesions on imaging. Radiographically, complex cystic lesions are reported more frequently than solid masses. ${ }^{2,6}$ Presence of a thick margin around the pancreatic lesion is indicative of pancreatic tuberculosis. ${ }^{7}$ Findings that may suggest mycobacterial infection include the presence of rim-enhanced lymph nodes in the peripancreatic region or the mesentery, ascites, and a thickened bowel wall in the ileocecal region. ${ }^{6,7}$ Criteria for the diagnosis of pancreatic tuberculosis were the presence of granuloma in histological sections or the presence of mycobacterium tuberculosis DNA by polymerase chain reaction (PCR). ${ }^{2}$ Most reported cases have been diagnosed via laparotomy. Percutaneous fine needle aspiration has been recently used to diagnose pancreatic tuberculosis. ${ }^{6}$

In conclusion, the importance of this case report is considering the suspicion of tuberculosis before or during the operative period, and appropriate bacteriological testing for these patients. In Islamic Republic of Iran, all physicians should be aware of the clinical features of pancreatic tuberculosis and conduct appropriate investigation to detect mycobacteria. It is also necessary to increase experience in endo-ultrasonography and fine needle aspiration in our country. Pancreatic tuberculosis is a treatable and a potentially curable condition. Thus, it is important for the physicians to consider this diagnosis especially in high-risk populations.

\section{CONFLICT OF INTEREST}

The authors declare no conflict of interest related to this work.

\section{REFERENCES}

1. Lotfi M, Lotfi K. Tuberculous Pancreatitis: Report of a "very rare case" and review of the literature.Ambassadors Online Magazines 2007;10, http: //ambassadors.net/archives/issue22/selectedstudy 2 .htm

2. Chatterjee S, Schmid ML, Anderson K, Oppong KW. Tuberculosis and the pancreas: a diagnostic challenge solved by endoscopic ultrasound. A case series. J Gastrointest Liver Dis 2012;21:105-7.

3. Knowles KF, Saltman D, Robson HG, Lalonde R. Tuberculous pancreatitis. Tubercle 1990;71:65-8. doi: 10.1016/0041-3879(90)90064-F.

4. Sotoudehmanesh R, Khatibian M, Zandi A, Shokri-
Shirvani J, Yaghoobi A, Sotoudeh M, et al. Pancreatic Tuberculosis Diagnosed with Endoscopic UltrasoundGuided Fine Needle Aspiration: Solid-Cystic Pancreatic Lesions in Endemic Areas. J Diag Medical Sonogr 2013;29:97-101. doi: 10.1177/8756479313476917.

5. Ladas SD, Vaidakis E, Lariou C, Anastasiou K, Chalevelakis G, Kintzonidis D, et al. Pancreatic tuberculosis in nonimmunocompromised patients: reports of two cases and a literature review. Eur J Gastroenterol Hepatol 1998;10:973-6.

6. Takhtani D, Gupta S, Suman K, Kakkar N, Challa $\mathrm{S}$, Wig JD, et al. Radiology of pancreatic tuberculosis: a report of three cases. Am J Gastroenterol 1996;91:1832-4.

7. Rezeig MA, Fashir BM, Al-Suhaibani H, Al-Fadda M, Amin T, Eisa H. Pancreatic tuberculosis mimicking pancreatic carcinoma: four case reports and review of the literature. Dig Dis Sci 1998;43:329-31. doi: 10.1023/A:1018854305652. 